\title{
Practical Land Grading Based ON LEAST SQUARES
}

\author{
By Edmar J. Scaloppi ${ }^{1}$ and Lyman S. Willardson, ${ }^{2}$ M. ASCE
}

\begin{abstract}
Aвstract: Practical methods for land grading design of a plane surface for rectangular and irregularly shaped fields based on a least squares analysis are presented. The least squares procedure leads to a system of three linear equations with three unknowns for determination of the best-fit plane. The equations can be solved by determinants (Cramer's rule) using a procedure capable of solution by many programmable calculators. The detailed computational process for determining the equation of the plane and a simple method to find the centroid location of an irregular field are also given. An illustrative example and design instructions are included to demonstrate the application of the design procedure.
\end{abstract}

\section{INTRODUCTION}

Land grading is basic to the design of an efficient and successful surface irrigation system. Land grading also is an effective method of providing adequate surface drainage, especially in intensive rainfall areas. Furthermore, proper land grading can be used to reduce erosion from rainfall.

Many of the current land grading computational techniques are limited to rectangular fields $(1,3,5,10)$, but not all fields requiring land grading are rectangular. The first land grading design method for irregularly shaped fields was developed by Chugg (2). He applied the least squares method to calculate the best fit slope, using transparent and coordinate papers to simplify and organize the computational process. The procedure requires the graphical determination of rectangular distances from the origin to the centroid and a separate determination of the centroid elevation. This method is considered to be too complicated to be generally adapted to land grading design (9).

Raju (6) proposed a fixed volume center method to calculate the slope of a graded soil surface plane. His method is based on the criterion that the total volume of earth and the center of volume will be the same before and after grading. This assumption is required to ensure a balance between cut and fill and to obtain the least amount of grading and movement of earth. Although the fixed volume center method has proven to be as accurate as other known methods of land grading (8), it is considered to be relatively difficult to use, and is time consuming when done by hand, especially for an irregularly shaped field. Shih and Kriz (7) concluded that the fixed volume center method is not as good as the least squares method, because it does not assume the same field surface shape before and after grading.

\footnotetext{
${ }^{1}$ Asst. Prof., Faculdade de Ciencias Agronomicas, UNESP, Botucatu, Sao Paulo, Brasil.

${ }^{2}$ Prof., Agr. and Irrigation Engr., Utah State Univ., Logan, UT.

Note.-Discussion open until October 1, 1986. To extend the closing date one month, a written request must be filed with the ASCE Manager of Journals. The manuscript for this paper was submitted for review and possible publication on November 19, 1985. This paper is part of the Journal of Irrigation and Drainage Engineering, Vol. 112, No. 2, May, 1986. CASCE, ISSN 0733-9437/86/0002-0098/ $\$ 01.00$. Paper No. 20576.
} 
In late 1971, Shih and Kriz (8) presented the symmetrical residuals method of land grading design for both rectangular and irregularly shaped fields. Their method is based on residual properties, Newton's divided difference interpolation procedure, and statistical properties of the best design with an unbiased estimate and minimum variance. Their comparisons with other known methods of design showed that the symmetrical residuals method is accurate and is easier to use than either Chugg's least squares method (2) or the fixed volume center method (6). However, the Symmetrical Residuals Method for land leveling is still considered too laborious for general use.

Sowell et al. (12) applied linear programming techniques to perform five land grading alternatives, and compared their results with those obtained by the symmetrical residuals method. They found that the linear programming technique always gave a smaller total sum of depths of cut but required significantly more computer time than the symmetrical residuals method. To the writers' knowledge, no attempt has yet been made to develop packaged computer routines to perform land grading designs using linear programming.

Recently, Manela (4) applied the least squares method to find the best fit plane in land grading design for both rectangular and irregularly shaped fields. Although his method gives accurate results and is not considered to be overly complicated, the computational process to determine the parameters of the equation of the plane can be simplified significantly.

The present work proposes simple and accurate analytical procedures for land grading design based on the least squares method. The procedures can be used on rectangular as well as irregular fields to get minimum earthwork volumes. The centroid location and elevation are not required in the computational process but are used in application of the method to meet alternate design specifications.

\section{Procedure}

The general equation of a plane surface is

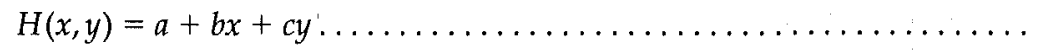

where $H=$ the elevation above a specified datum of any point on the plane; $a=$ the elevation above a specified datum of the origin of the coordinate system; $b$ and $c=$ the slopes of the plane in the $X$ and $Y$ coordinate directions, respectively; and $x$ and $y=$ the coordinates of the point in the $X$ and $Y$ directions, respectively.

In a land grading situation, when the equation of a particular plane is known, the vertical distance between the original ground elevation and the elevation at any corresponding point on the plane is determined by

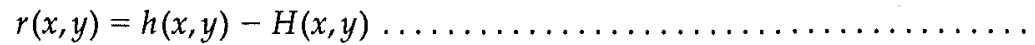

where $r=$ the vertical distance between the original ground elevation $h$ and the elevation $H$ for a given point on the plane with the coordinates $x$ and $y$. Hence the sum of the squares $R$ of the vertical distances $r$ for any number of points is 


$$
R=\sum_{x=1}^{m} \sum_{y=1}^{n} r\left(x_{i}, y_{j}\right)^{2}=\sum_{x=1}^{m} \sum_{y=1}^{n}\left[h\left(x_{i}, y_{j}\right)-H\left(x_{i}, y_{j}\right)\right]^{2}
$$

where $m$ and $n=$ the number of $x$ and $y$ coordinate points on the plane, respectively.

Substituting Eq. 1 into Eq. 3 gives

$$
R=\sum_{x=1}^{m} \sum_{y=1}^{n}\left[h\left(x_{i}, y_{j}\right)-\left(a+b x_{i}+c y_{j}\right)\right]^{2}
$$

In the method of least squares, the values of $a, b$, and $c$ are needed such that $R$ is a minimum. A necessary condition for $R$ to be a minimum is that the partial derivatives of $R$ with respect to $a, b$, and $c$ are zero. The details of the least squares development are shown in Appendix I.

\section{Application of the Procedure}

Data from Chugg (2) are used here to illustrate the procedure for finding the plane surface of best fit for an irregularly shaped field and to illustrate the use of the least squares plane in landgrading design. The relative elevations of the field surface are given in Fig. 1, which also shows the rectangular grid system used in the computations and the area weighting factors needed for the computation of cut and fill volumes.

The computations are all performed considering unit distances and unit areas according to the proposed procedure. At the end of the computations, the appropriate actual field dimensions can be used to com-

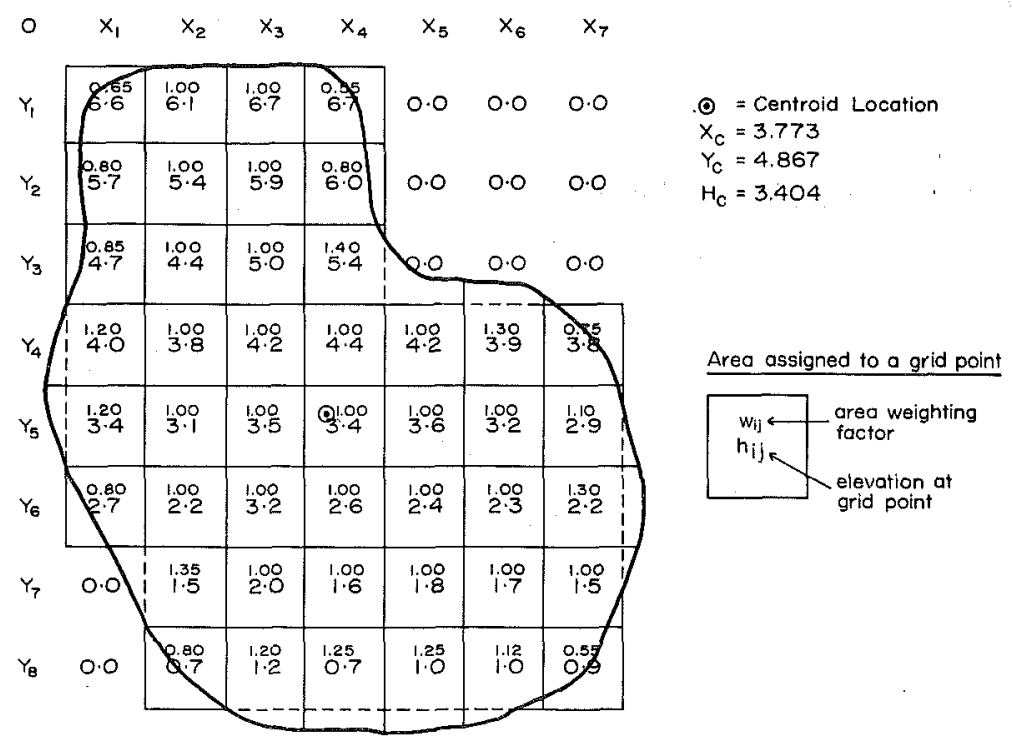

FIG. 1.- Rectangular Grid System, Elevations, and Area Weighting Factors for Land Grading Irregular Fleld 
pute the volumes of cuts and fills. The field and office procedure followed is outlined in detail:

1. Stake the field in a regular grid pattern. Measure the distances from the last grid point in each line to the edge of the field in both directions in order to define the field boundaries.

2. Determine the elevation of each grid point and the elevation of the soil surface at the end of each grid line projected to the edge of the field. This latter information is not used in the computations but is needed to mark cuts and fills at the edges of the field for the benefit of the machine operators when the actual grading is done.

3. Draw a plan map of the field and sketch the field boundaries, (Fig. 1).

4. At each grid point, write the elevation, using the grid point itself as the decimal point in the elevation number (Fig. 1).

5. Write a weighting number for each grid point above the elevation of the grid point to indicate the area weighting factor for that grid point. Interior regular grid points have a weighting factor of 1.00. Grid points on the boundary will have area weighting factors greater or less than 1.00 depending on the amount of surface area represented by the grid point. For areas outside regular grid dimensions that could be represented by more than one grid point, add the extra area to the grid point in the direction of least slope, (Fig. 1).

6. Assign zero elevations for grid points that fall outside the field boundaries (Fig. 1).

7. Using the least squares method, compute the equation of the least squares plane, and the coordinates and elevation of the centroid. Any grid point with a zero elevation is not included in the calculation.

The equations shown in Appendix I have been simplified and reorganized below for simplified computation:

$$
\begin{aligned}
& A_{1}=\sum_{x=1}^{7} \sum_{y=1}^{8}=45=N=\text { actual number of grid points in the field } \ldots \ldots \\
& A_{2}=B_{1}=\sum_{x=1}^{7} \sum_{y=1}^{8} x_{i}=(1 \times 6)+(2 \times 8)+\ldots+(7 \times 5)=168 \ldots \ldots \ldots \\
& \qquad A_{2}=(a \times b)+(c \times d)+\ldots+(e \times f)=168
\end{aligned}
$$




$$
B_{2}=\sum_{x=1}^{7} \sum_{y=1}^{8} x_{i}^{2}=\left(1^{2} \times 6\right)+\left(2^{2} \times 8\right)+\ldots+\left(7^{2} \times 5\right)=788
$$

$$
B_{2}=(a \times b)+\ldots+(c \times d)=788
$$

$$
\begin{array}{ll}
a=x_{1}^{2} & c=x_{7}^{2} \\
b=\text { number of } y \text { values at } x_{1} & d=\text { number of } y \text { values at } x_{7}
\end{array}
$$

$$
\begin{aligned}
& C_{3}=\sum_{y=1}^{8} \sum_{x=1}^{7} y_{j}^{2}=\left(1^{2} \times 4\right)+\left(2^{2} \times 4\right)+\ldots+\left(8^{2} \times 6\right)=1,273 \\
& C_{3}=(a \times b)+\ldots+(c \times d)=1,273
\end{aligned}
$$

$$
\begin{aligned}
B_{3}=C_{2}=\sum_{x=1}^{7} \sum_{y=1}^{8} x_{i} y_{j} & =(1 \times 1)+(1 \times 2)+\ldots+(7 \times 8)=885 \ldots \ldots \\
B_{3} & =(a \times b)+\ldots+(c \times d)=885
\end{aligned}
$$

$$
\begin{aligned}
a & =x_{1} \quad c=x_{7} \\
b & =y_{1} \quad d=y_{8} \\
F_{1} & =\sum_{x=1}^{7} \sum_{y=1}^{8} h\left(x_{i}, y_{j}\right)=6.6+5.7+\ldots+0.9=153.2
\end{aligned}
$$

$$
\begin{aligned}
& F_{1}=(a)+(b)+\ldots+(c)=153.2 \\
& a=h\left(x_{1}, y_{1}\right) \quad c=h\left(x_{7}, y_{8}\right) \\
& b=h\left(x_{1}, y_{2}\right) \\
& F_{2}=\sum_{x=1}^{7} \sum_{y=1}^{8}\left[(x) h\left(x_{i}, y_{j}\right)\right]=(1 \times 6.6)+(1 \times 5.7)+\ldots+(7 \times 0.9)=516.5 \ldots \\
& F_{2}=(a \times b)+(c \times d)+\ldots+(e \times f)=516.5 \\
& a=x_{1} \quad d=h\left(x_{1}, y_{2}\right) \\
& b=h\left(x_{1}, y_{1}\right) \quad e=x_{7} \\
& c=x_{1} \quad f=h\left(x_{7}, y_{8}\right) \\
& F_{3}=\sum_{y=1}^{8} \sum_{x=1}^{7}\left[(y) h\left(x_{i}, y_{j}\right)\right]=(1 \times 6.6)+(1 \times 6.1)+\ldots+(8 \times 0.9)=579.6 \ldots \\
& F_{3}=(a \times b)+(c \times d)+\ldots+(e \times f)=579.6 \\
& a=y_{1} \quad d=h\left(x_{2}, y_{1}\right) \\
& b=h\left(x_{1}, y_{1}\right) \quad e=y_{8} \\
& c=y_{1} \quad f=h\left(x_{7}, y_{8}\right)
\end{aligned}
$$

The simplified computations for the least squares plane equation using Cramer's Rule is 
$D=A_{1} B_{2} C_{3}+A_{3} B_{1} C_{2}+A_{2} B_{3} C_{1}-A_{3} B_{2} C_{1}-A_{2} B_{1} C_{3}-A_{1} B_{3} C_{2}$

$D_{1}=F_{1} B_{2} C_{3}+F_{3} B_{1} C_{2}+F_{2} B_{3} C_{1}-F_{3} B_{2} C_{1}-F_{2} B_{1} C_{3}-F_{1} B_{3} C_{2} \ldots$

$D_{2}=A_{1} F_{2} C_{3}+A_{3} F_{1} C_{2}+A_{2} F_{3} C_{1}-A_{3} F_{2} C_{1}-A_{2} F_{1} C_{3}-A_{1} F_{3} C_{2} \ldots$

$D_{3}=A_{1} B_{2} F_{3}+A_{3} B_{1} F_{2}+A_{2} B_{3} F_{1}-A_{3} B_{2} F_{1}-A_{2} B_{1} F_{3}-A_{1} B_{3} F_{2} \ldots$

$a=\frac{D_{1}}{D}=7.3254 \quad b=\frac{D_{2}}{D}=0.01049 \quad c=\frac{D_{3}}{D}=0.7976 \ldots \ldots \ldots$

which gives the following equation:

$H(x, y)=7.3254-0.01049 x-0.79762 y$

The centroid location in the field is easily determined by

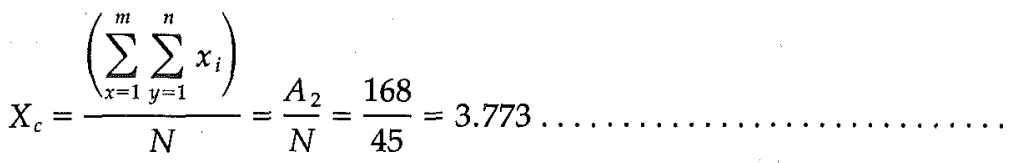

$$
\begin{aligned}
& Y_{c}=\frac{\left(\sum_{x=1}^{m} \sum_{y=1}^{n} y_{j}\right)}{N}=\frac{A_{3}}{N}=\frac{219}{45}=4.867
\end{aligned}
$$

The $x$ and $y$ distances computed are the number of unit grid spaces. The values computed above are exactly the same as those obtained by Chugg (2).

The elevation of the least squares plane at the centroid is found by substituting the values of $x_{c}$ and $y_{c}$ in Eq. 19 to determine $H_{c}=3.404$ (Fig. 1).

8. For each grid point, compute the elevation of the least squares plane using the equation of the plane. Subtract the plane elevation at the grid point from the field elevation at the grid point and multiply the difference by the area weighting factor for that grid point. Positive differences are accumulated as volumes of cut. Negative differences are accumulated as volumes of fill. The volume of the cuts should be an average of about $15 \%$ greater than the volume of the fills to account for shrinkage that occurs during earth moving. The percent difference between cut and fill volumes varies with soils. Change the value of $a$, down to increase the volume of cuts, or up to increase the volume of fills to get the required proportional balance between cuts and fills. When the computations are complete, use the equation of the final plane with the appropriate $x$ and $y$ coordinates to check critical elevations at the edges of the field to make sure that the plane is not too high or too low and to mark the cuts and fills at the boundaries of the field.

9. To remove any cross-slope in the field, it is necessary to make $b$ equal to zero and to force the plane to be horizontal in the $x$ direction or make $c$ equal to zero to force the plane to be horizontal in the $y$ direction. Solve for new values of $a$ and $c$, or $a$ and $b$ as appropriate, using the coordinates and elevation of the centroid and two appropriate elevations and grid point locations of the plane at the upper edge of the 
field. Any plane that goes through the centroid will give the least volume cuts and fills for any specified position of the plane but will not give the minimum earth moving volume, which only occurs for the least squares plane. Change $a$ to balance the cuts and fills. Check critical elevations at the edges.

Since 3 points determine a plane, the 3 points used to make a plane horizontal in either direction can be put into Eq. 1 and the solution found using Cramer's Rule. Including the centroid coordinates and elevation of the least squares plane as one of the points guarantees the minimum amount of earthwork for the chosen plane. The system of simultaneous equations for a plane passing through any 3 points is

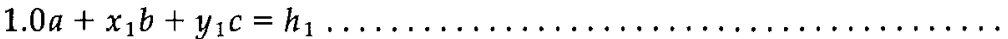

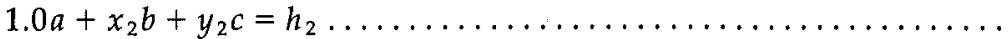

and the Cramer's Rule Solution is

$$
\begin{aligned}
& d=x_{2} y_{3}+x_{1} y_{2}+x_{3} y_{1}-x_{2} y_{1}-x_{3} y_{2}-x_{1} y_{3} \ldots \ldots \ldots \ldots \ldots \ldots \ldots \\
& d_{1}=h_{1} x_{2} y_{3}+h_{3} x_{1} y_{2}+h_{2} x_{3} y_{1}-h_{3} x_{2} y_{1}-h_{1} x_{3} y_{2}-h_{2} x_{1} y_{3} \ldots \ldots \ldots \\
& d_{2}=h_{2} y_{3}+h_{1} y_{2}+h_{3} y_{1}-h_{2} y_{1}-h_{3} y_{2}-{ }_{1} h_{1} y_{3} \\
& d_{3}=h_{3} x_{2}+h_{2} x_{1}+h_{1} x_{3}-h_{1} x_{2}-h_{2} x_{3}-h_{1} h_{3} \\
& a=\frac{d_{1}}{d} \quad b=\frac{d_{2}}{d} \quad c=\frac{d_{3}}{d}
\end{aligned}
$$

10. To level to a control point, use the centroid position and elevation plus the elevation and location of the control point and one other point to determine the values of $a, b$, and $c$ using Eqs. 22-29 and 1. Change $a$ to balance cuts and fills. Check the final plane elevations at points on the edge of the field to determine whether the final plane position is satisfactory.

11. To level to a control edge, use the centroid position and elevation plus the position and elevation of two points on the control edge to determine $a, b$, and $c$ using Eqs. 22 to 29 and 1. If cuts and fills do not balance, recalculate $a, b$, and $c$ by raising or lowering the elevation of the plane at the centroid location until the cuts and fills are balanced. Check the edge elevations with the final equations.

The procedures outlined above are amenable to calculation by hand calculator, programmable calculator or computer. Each data point has 4 dimensions: (1) $h_{i j}=$ the original ground elevation of a point in the field; (2) $x_{i}=$ the coordinate of the point in the $x$ direction; (3) $y_{j}=$ the coordinate of the point in the $y$ direction; and (4) $W_{i j}=$ the relative area weighting factor for the area represented by each grid point. By assigning a zero elevation to grid points outside the area to be graded, the zero elevation can be used to logically reject points that should not be included in the computations. 
12. The final volume of earthwork is obtained by multiplying the number obtained in Step 8 above for cuts (positive accumulation) and for fills (negative accumulation) by the area represented by a normal grid point. If the elevations are in feet and the grid points are $100 \mathrm{ft}$ apart, the area represented by each grid point would be $10,000 \mathrm{sq} f \mathrm{ft}$ and the computed volumes of cut and fill will be in cubic feet. Since the computations use nondimensional or relative values, any appropriate units can be used.

13. For dead-level land grading, the centroid elevation can be used as a starting elevation for properly balancing cut and fill volumes. The procedures of items 8 and 12 apply following determination of the centroid elevation.

\section{Summary and Conclusions}

Practical procedures for using a least squares analysis in land grading computations have been presented. Most of the land grading single plane situations that may occur in irrigation practice have been covered for a single plane system. Methods for fitting minimum earthwork planes to specific site requirements are included. The methods presented are amenable to computation by calculators or computers.

\section{ACKNOWLEDGMENTS}

The study in this paper is in connection with a project of the National Center of Agricultural Engineering-CENEA, Sorocaba, SP, Brazil.

\section{Appendix I.-Least Squares Solution}

The following presentation of the details of development of the least squares solution has been reduced to the ultimate useful solution shown in the text. The development begins with Eq. 4 (shown earlier) and continues to the final forms of the equation that can be solved by determinants and Cramer's Rule.

Eq. 4, repeated here, is the equation for the sum of squares of the distances between the soil surface at individual grid points within a field to be graded and a plane described by Eq. 1 (also first shown earlier in the test):

Eq. $1 \quad H(x, y)=a+b x+c y$

Eq. $4 \quad R=\sum_{x=1}^{m} \sum_{y=1}^{n}\left[h\left(x_{i}, y_{j}\right)-\left(a+b x_{i}+c y_{j}\right)\right]^{2}$

where $R=$ the sum of squares of the elevation differences; $m$ and $n=$ the number of points in the $x$ and $y$ directions, respectively, of a rectangular grid enclosing the area to be graded; and $h=$ the elevation of the soil surface at an interior grid point $\left(x_{i}, y_{j}\right)$. The parameter $a$ is the elevation of the plane of Eq. 1 at the origin of the coordinate system, $b$ is the slope of the plane in the $x$ direction, and $c$ is the slope of the plane in the $y$ direction. 
A necessary condition for $R$ to be a minimum is that its partial derivatives with respect to $a, b$, and $c$ be equal to zero.

$$
\begin{aligned}
& \frac{\partial R}{\partial a}=-2 \sum_{x=1}^{m} \sum_{y=1}^{n}\left[h\left(x_{i}, y_{j}\right)-\left(a+b x_{i}+c y_{j}\right)\right]^{2}=0 \ldots \ldots \ldots \ldots \ldots \ldots \\
& \frac{\partial R}{\partial b}=-2 \sum_{x=1}^{m} \sum_{y=1}^{m}(x)[h(x, y)-(a+b \times c y)]=0 \\
& \frac{\partial R}{\partial c}=-2 \sum_{x=1}^{m} \sum_{y=1}^{m}(y)[h(x, y)-(a+b x+c y)]=0
\end{aligned}
$$

The partial derivatives of Eqs. 30-32 result in a series of 3 equations with 3 unknowns $a, b$, and $c$ that can be solved by determinants and Cramer's Rule:

$$
\begin{aligned}
& a\left(\sum_{x=1}^{m} \sum_{y=1}^{n} x_{i}\right)+b\left(\sum_{x=1}^{m} \sum_{y=1}^{n} x_{i}\right)+c\left(\sum_{x=1}^{m} \sum_{y=1}^{n} y_{j}\right)=\sum_{x=1}^{m} \sum_{y=1}^{n} h\left(x_{i}, y_{j}\right) \ldots \ldots \\
& a\left(\sum_{x=1}^{m} \sum_{y=1}^{n} x\right)+b\left(\sum_{x=1}^{m} \sum_{y=1}^{n} x^{2}\right)+c\left(\sum_{x=1}^{m} \sum_{y=1}^{n} x y\right)=\sum_{x=1}^{m} \sum_{y=1}^{n}(x)\left[h\left(x_{i} y_{j}\right)\right] \ldots \\
& a\left(\sum_{y=1}^{n} \sum_{x=1}^{m} y\right)+b\left(\sum_{x=1}^{m} \sum_{y=1}^{n} x y\right)+c\left(\sum_{y=1}^{n} \sum_{x=1}^{m} y_{i}^{2}\right)=\sum_{x=1}^{m} \sum_{y=1}^{n}(y)\left[h\left(x_{i} y_{j}\right)\right] \ldots
\end{aligned}
$$

The determinant of this system of equations with 3 unknowns $a, b$, and $c$ is not zero, so the equations have exactly 1 solution. Some programmable calculators have packaged or internal programs that can perform the computations for a determinant solution from the resulting elements of the matrix and the column vectors.

The solution of the system of Eqs. 33-35 given below follow the Cramer's Rule Formulas:

$a=\frac{D_{1}}{D} ; \quad b=\frac{D_{2}}{D} ; \quad c=\frac{D_{3}}{D}$.

in which $D=$ the determinant of the system of 3 linear equations (Eqs. 33-35); and $D_{k}(K=1,2$, and 3$)=$ the determinant obtained by replacing the $K$ th column in $D$ with the column vector. The detailed equations for computing $D$ and $D_{k}(K=1,2$, and 3) from the grid data are given below. The value of $N$ in Eq. $8 a$ is the actual number of data points within the area to be graded. The number of points may be much larger. Only the points $x_{i}$ and $y_{j}$, which are within the field to be graded, should be used in the computations:

$$
\begin{aligned}
& D=(N)\left(\sum_{x=1}^{m} \sum_{y=1}^{n} x_{i}^{2}\right)\left(\sum_{x=1}^{m} \sum_{y=1}^{n} y_{i}^{2}\right)-\left(\sum_{x=1}^{m} \sum_{y=1}^{n} x y_{j}\right)^{2} \\
& +2\left(\sum_{x=1}^{m} \sum_{y=1}^{n} x_{i}\right)\left(\sum_{x=1}^{m} \sum_{y=1}^{n} y_{j}\right)\left(\sum_{x=1}^{m} \sum_{y=1}^{n} x_{i} y_{j}\right)
\end{aligned}
$$




$$
\begin{aligned}
& -\left(\sum_{x=1}^{m} \sum_{y=1}^{n} x^{2}\right)\left(\sum_{y=1}^{n} \sum_{x=1}^{m} y\right)^{2}-\left(\sum_{y=1}^{n} \sum_{x=1}^{m} y^{2}\right)\left(\sum_{x=1}^{m} \sum_{y=1}^{n} x\right)^{2} \\
& D_{1}=\sum_{x=1}^{m} \sum_{y=1}^{n} h(x, y)\left(\sum_{x=1}^{m} \sum_{y=1}^{n} x^{2}\right)\left(\sum_{y=1}^{n} \sum_{x=1}^{m} y^{2}\right)-\left(\sum_{x=1}^{m} \sum_{y=1}^{n} x y\right)^{2} \\
& +\sum_{x=1}^{m} \sum_{y=1}^{n} x h(x, y)\left(\sum_{y=1}^{n} \sum_{x=1}^{m} y\right)\left(\sum_{x=1}^{m} \sum_{y=1}^{n} x y\right)-\left(\sum_{x=1}^{m} \sum_{y=1}^{n} x\right)\left(\sum_{y=1}^{n} \sum_{x=1}^{m} y^{2}\right) \\
& +\sum_{y=1}^{n} \sum_{x=1}^{m} y h(x, y)\left(\sum_{x=1}^{m} \sum_{y=1}^{n} x\right)\left(\sum_{x=1}^{m} \sum_{y=1}^{n} x y\right)-\left(\sum_{y=1}^{n} \sum_{x=1}^{m} y\right)\left(\sum_{x=1}^{m} \sum_{y=1}^{n} x^{2}\right) \\
& D_{2}=\sum_{x=1}^{m} \sum_{y=1}^{n} h(x, y)\left(\sum_{y=1}^{n} \sum_{x=1}^{m} y\right)\left(\sum_{x=1}^{m} \sum_{y=1}^{n} x y\right)-\left(\sum_{x=1}^{m} \sum_{y=1}^{n} x\right)\left(\sum_{y=1}^{n} \sum_{x=1}^{m} y^{2}\right) \\
& +\sum_{x=1}^{m} \sum_{y=1}^{n} x h(x, y) N\left(\sum_{y=1}^{n} \sum_{x=1}^{m} y^{2}\right)-\left(\sum_{y=1}^{n} \sum_{x=1}^{m} y\right)^{2} \\
& +\sum_{y=1}^{n} \sum_{x=1}^{m} y h(x, y)\left(\sum_{x=1}^{m} \sum_{y=1}^{n} x\right)\left(\sum_{y=1}^{n} \sum_{x=1}^{m} y\right)-N\left(\sum_{x=1}^{m} \sum_{y=1}^{n} x y\right) \\
& D_{3}=\sum_{x=1}^{m} \sum_{y=1}^{n} h\left(x_{i}, y_{j}\right)\left(\sum_{x=1}^{m} \sum_{y=1}^{n} x\right)\left(\sum_{x=1}^{m} \sum_{y=1}^{n} x y\right)-\left(\sum_{y=1}^{n} \sum_{x=1}^{m} y\right)\left(\sum_{x=1}^{m} \sum_{y=1}^{n} x^{2}\right) \\
& +\sum_{x=1}^{m} \sum_{y=1}^{n} x h(x, y)\left(\sum_{x=1}^{m} \sum_{y=1}^{n} x\right)\left(\sum_{y=1}^{n} \sum_{x=1}^{m} y\right)-N\left(\sum_{x=1}^{m} \sum_{y=1}^{n} x y\right) \\
& +\sum_{y=1}^{n} \sum_{x=1}^{m} y h(x, y) N\left(\sum_{x=1}^{m} \sum_{y=1}^{n} x^{2}\right)-\left(\sum_{x=1}^{m} \sum_{y=1}^{n} x\right)^{2}
\end{aligned}
$$

In Eqs. 37-40 there are a number of recurring factors that can be identified as given below:

$$
\begin{aligned}
& C_{1}=\left(\sum_{x=1}^{m} \sum_{y=1}^{n} x_{i}^{2}\right)\left(\sum_{x=1}^{m} \sum_{y=1}^{n} y_{j}^{2}\right)-\left(\sum_{x=1}^{m} \sum_{y=1}^{n} x y\right)^{2} \ldots \ldots \ldots \\
& C_{2}=\left(\sum_{y=1}^{n} \sum_{x=1}^{m} y\right)\left(\sum_{x=1}^{m} \sum_{y=1}^{n} x y\right)-\left(\sum_{x=1}^{m} \sum_{y=1}^{n} x\right)\left(\sum_{y=1}^{n} \sum_{x=1}^{m} y^{2}\right) \ldots \\
& C_{3}=\left(\sum_{x=1}^{m} \sum_{y=1}^{n} x\right)\left(\sum_{x=1}^{m} \sum_{y=1}^{n} x y\right)-\left(\sum_{y=1}^{n} \sum_{x=1}^{m} y\right)\left(\sum_{x=1}^{m} \sum_{y=1}^{n} x^{2}\right) \ldots \\
& C_{4}=\left(\sum_{x=1}^{m} \sum_{y=1}^{n} x\right)\left(\sum_{y=1}^{n} \sum_{x=1}^{m} y\right)-N\left(\sum_{x=1}^{m} \sum_{y=1}^{n} x y\right) \ldots \ldots
\end{aligned}
$$

Therefore, the equations for the determinants for the purposes of com- 
putation can be simplified to those which follow. It should again be emphasized that $N$ is the number of points within the actual area to be graded and is not the number of grid points in the $x, y$ grid system. Only the data points within the area to be graded should be used in the computation. One method of avoiding difficulties is to assign zero elevation values to grid points outside the area to be graded so they can be identified and eliminated from the computations.

The useful equations for determining the values of $a, b$, and $c$ for the equation of the least squares plane (Eq. 1) from Eq. 36 are

$$
\begin{aligned}
& D=N C_{1}+2\left(\sum_{x=1}^{m} \sum_{y=1}^{n} x_{i}\right)\left(\sum_{x=1}^{m} \sum_{y=1}^{n} y_{j}\right)\left(\sum_{x=1}^{m} \sum_{y=1}^{n} x_{i} y_{j}\right) \\
& -\left(\sum_{x=1}^{m} \sum_{y=1}^{n} x^{2}\right)\left(\sum_{y=1}^{n} \sum_{x=1}^{m} y\right)^{2}-\left(\sum_{y=1}^{n} \sum_{x=1}^{m} y^{2}\right)\left(\sum_{x=1}^{m} \sum_{y=1}^{n} x\right)^{2} \ldots \ldots \ldots \ldots \ldots \\
& D_{1}=\sum_{x=1}^{m} \sum_{y=1}^{n} h(x, y) C_{1}+\sum_{x=1}^{m} \sum_{y=1}^{n} x h(x, y) C_{2}+\sum_{y=1}^{n} \sum_{x=1}^{m} y h(x, y) C_{3} \ldots \ldots \ldots \\
& D_{2}=\sum_{x=1}^{m} \sum_{y=1}^{n} h(x, y) C_{2}+\sum_{x=1}^{m} \sum_{y=1}^{n} x h(x, y) N\left(\sum_{y=1}^{n} \sum_{x=1}^{m} y^{2}\right) \\
& -\left(\sum_{y=1}^{n} \sum_{x=1}^{m} y\right)^{2}+\sum_{y=1}^{n} \sum_{x=1}^{m} y h(x, y) C_{4} \ldots \ldots \ldots \ldots \\
& D_{3}=\sum_{x=1}^{m} \sum_{y=1}^{n} h(x, y) C_{3}+\sum_{x=1}^{m} \sum_{y=1}^{n} x h(x, y) C_{4} \\
& +\sum_{y=1}^{n} \sum_{x=1}^{m} y h(x, y) N\left(\sum_{x=1}^{m} \sum_{y=1}^{n} x^{2}\right)-\left(\sum_{x=1}^{m} \sum_{y=1}^{n} x\right)^{2} \ldots \ldots \ldots \ldots \ldots
\end{aligned}
$$

When the parameters of the least squares plane equation have been determined, the equation of the plane can be used in the land grading design.

\section{AppendiX II.-References}

1. Butler, E. D., "The Land Leveling Program in the Arkansas Delta," Agricultural Engineering, Vol. 42, No. 3, Mar., 1961, pp. 128-131.

2. Chugg, G. E., "Calculations for Lang Gradation," Agricultural Engineering, Vol. 28, No. 10, Oct., 1947, pp. 461-463.

3. Givan, C. V., "Land Grading Calculations," Agricultural Engineering, Vol. 21, No. 1, Jan., 1940, pp. 11-12.

4. Manela, M., "Construcao de planos geometricos em sistematizacao de solos pelo metodo dos minimos quadrados generalizados," Irrigacao e Tecnologia Moderna, Vol. 10, 1983, pp. 32-34, 36-37.

5. Marr, J. C., "Grading Land for Surface Irrigation," Division of Agricultural Sciences, Univ. of California, Circular 438, 1957, 55 pp.

6. Raju, V. S., "Land Grading for Irrigation," Transactions of the American Society of Agricultural Engineers, Vol. 3, No. 1, Jan.-Feb., 1960.

7. Shih, S. F., and Kriz, G. J., "Comparison of Types and Methods of Land 
Forming Design," Transactions of the American Society of Agricultural Engineers, Vol. 14, No. 5, Sept.-Oct., 1971, pp. 990-994.

8. Shih, S. F., and Kriz, G. J., "Symmetrical Residuals Method for Land Forming Design," Transactions of the American Society of Agricultural Engineers, Vol. 14, No. 6; Nov.-Dec., 1971, pp. 1195-1200.

9. Shih, S. F., Kriz, G. J., and Sowell, R. S., "Land Forming Design for NonRectangular Fields," Transactions of the American Society of Agricultural Engineers, Vol. 16, No. 4, July-Aug., 1973, pp. 810-813.

10. Smerdon, E. T., Tefertiller, K. R., Kilmer, R. E., and Billingsley, R. V., "Electronic Computers for Least-Cost Land-Forming Calculations," Transactions of the American Society of Agricultural Engineers, Vol. 9, No. 2, Mar.-Apr., 1966, pp. 190-193.

11. Soil Conservation Service, SCS National Engineering Handbook, Section 15, Chapter, 12, Land Leveling, USDA, Washington, D.C., 1970, 59 pp.

12. Sowell, R. S., Shih, S. F., and Kriz, G. J., "Land Forming Design by Linear Programming," Transactions of the American Society of Agricultural Engineers, Vol. 16, No. 3, May-June, 1973, pp. 296-301.

\section{APPENDIX III.-NOTATION}

The following symbols are used in this paper:

$$
\begin{aligned}
a= & \begin{array}{l}
\text { elevation of plane surface above specified datum at origin } \\
\text { of coordinate system; }
\end{array} \\
b= & \text { slope of plane in } X \text { coordinate direction; } \\
c= & \text { slope of plane in } Y \text { coordinate direction; } \\
\text { elevation of plane above specified datum for a point on plane } & \text { with coordinates } x \text { and } y ; \\
h(x, y) & \text { original ground elevation for a point on field surface with } \\
& \text { coordinates } x \text { and } y ; \\
N= & \text { number of field points with elevation data; } \\
R= & \text { sum of squares of vertical distances between original ground } \\
& \text { elevations and grade elevations for all grid points of field; } \\
r(x, y)= & \text { vertical distance between original ground elevation and grade } \\
& \text { elevation for a point with coordinates } x \text { and } y ; \\
W_{i j}= & \text { relative area weighting factor for area represented by a grid } \\
& \text { point; } \\
x= & \text { coordinate of a point in } X \text { direction; and } \\
y= & \text { coordinate of a point in } Y \text { direction. }
\end{aligned}
$$

